\title{
Salivary Cortisol Levels in Students Challenged with a Testing Stressor
}

\author{
KATHLEEN KENWRIGHT, PATTY W LIDDELL, LEONARD BLOOM, AUDREY ZUCKER-LEVIN, \\ ANN H NOLEN, LAWRENCE W FAULKNER, ROSEMARY E BATORSKI
}

OBJECTIVE: The objective was twofold. The focus of the study was primarily to determine if the stress of a particularly difficult exam could cause students to lose the normal diurnal variation seen in human cortisol levels and secondarily, to validate the use of a competitive enzyme immunoassay for salivary cortisol.

DESIGN: Physical therapy students enrolled in Research Design were asked to participate in the study by collecting baseline evening and morning salivary cortisols during what was regarded as a relatively stress free time in the Fall of 2009. The following spring, the same students were asked for samples the evening before and morning of their first Kinesiology test, traditionally a stressful time. Method validation was accomplished using instrumentation owned by the Medical Laboratory Science (MLS) Program and analysis was performed by MLS faculty and a second year MLS student.

SETTING: Participants were enrolled in the College of Health Sciences at the University of Tennessee in Memphis. Sample collection and testing was performed in the student laboratory of the Medical Laboratory Science Program.

PARTICIPANTS: Physical therapy students in their first year of a three-year entry level doctorate program, DPT.

RESULTS: This group of students did not lose their diurnal variation of cortisol. However, an unexpected finding was noted: the students' salivary cortisol specimen collected in the morning of the fall semester was significantly higher than the salivary cortisol specimen collected the morning of the test in the spring semester $(\mathrm{p}=.019)$. Method validation was successful demonstrating a strong correlation $(r=0.915)$ when compared to the reference laboratory.
CONCLUSIONS: Cortisol diurnal variation was not lost in the study participants, but further studies should be performed due to the low percentage of students completing the study and the lack of demographic diversity. Even though the method validation in the student laboratory setting demonstrates that it is indeed possible to obtain the same excellent correlation as is seen in a clinical setting, the student laboratory is not CLIA certified, so assays can be performed for research use only.

ABBREVIATIONS: ACTH-adrenocorticotropic hormone, CRH-corticotrophin-releasing hormone, DPTDoctorate in Physical Therapy, BS-Baccalaureate of Science, BA-Baccalaureate of Arts, IRB-Internal Review Board, GPA-Grade Point Average, EIA-Enzyme Immunoassay, BMI-Basic Metabolic Index, CLIAClinical Laboratory Improvement Amendments, NDnone detected

INDEX TERMS: stress, cortisol, exams, students

Clin Lab Sci 2011;24(4):221

Kathleen Kenwright, MS, MT,(ASCP)SI,MB ${ }^{C M}$, University of Tennessee Health Science Center, Memphis, TN 38163.

Patty W. Liddell MS, MT(ASCP)SH, Baptist College of Health Sciences, Memphis, TN 38163

Leonard Bloom MPH, CT(ASCP), The University of Tennessee Health Science Center, College of Allied Health Sciences, Memphis, TN.

Audrey Zucker-Levin PT, PhD, MBA, GCS, The University of Tennessee Health Science Center, College of Allied Health Sciences, Memphis, TN. 


\section{RESEARCH AND REPORTS}

Ann H. Nolen, Psy.D., OTR, FAOTA, The University of Tennessee Health Science Center, College of Allied Health Sciences, Memphis, TN.

Lawrence W. Faulkner, Ph.D. OT/L, The University of Tennessee Health Science Center, College of Allied Health Sciences, Memphis, TN.

Rosemary E. Batorski, MEd, MOT, OTR, The University of Tennessee Health Science Center, College of Allied Health Sciences, Memphis, TN.

Address for Correspondence: Kathleen Kenwright, MS, $M T,(A S C P) S I, M B^{C M}$, University of Tennessee Health Science Center, 930 Madison Avenue, Suite 672, Memphis, TN 38163,901-448-6338, kkenwrig@uthsc. edu

\section{INTRODUCTION}

Cortisol is a steroid hormone produced by the adrenal glands in response to adrenocorticotropic hormone (ACTH) secreted by the pituitary. ${ }^{1}$ The typical diurnal variation of cortisol secretion has been well established. Cortisol shows an increase in the early morning hours, peaking at or slightly before the time of waking, and decreases in the evening. ${ }^{2}$ This hormone plays an important role in maintaining homeostasis by affecting multiple organ systems and processes. Under stressful situations, the body responds by increasing the production of cortisol by initiating a series of events involving the hypothalamus, pituitary and adrenal cortex called the HPA axis. Specifically, corticotrophinreleasing hormone $(\mathrm{CRH})$ released by the hypothalamus triggers release of adrenocorticotropic hormone (ACTH) from the pituitary which in turn causes the secretion of glucocorticoids from the adrenal cortex. ${ }^{3}$ The strength of the stimulus causes some variation in circadian peak levels and the normal variation has been shown to be disrupted during times of stress. ${ }^{2}$

\section{Effect on memory}

Though elevated cortisol in response to stress is a natural response, it is sometimes detrimental for students in that some forms of memory are impaired. There is abundant evidence that the secretion of glucocorticoid stress hormones may modulate memory functioning. ${ }^{4}$ The frontal lobe and hippocampus in humans contain a high density of glucocorticoid receptors and are areas for cognition and emotion.
During times of stress there may be a pronounced deficit in working memory but at the same time, enhancements may be seen associated with other forms of memory. ${ }^{5}$

\section{MATERIAL AND METHODS \\ Multidisciplinary Project}

Four departments within the College of Allied Health at the University of Tennessee Health Science Center participated in various aspects of this project. The following outlines some goals anticipated by the faculty of the departments involved in the study.

The purposes of this multidisciplinary project were to:

- Validate salivary cortisol in our Clinical Laboratory Science (MLS) student laboratory.

- Determine if the stress of an exam would cause students to lose their normal diurnal variation of cortisol.

- Determine if there is a correlation between cortisol concentration and a test grade.

- Determine if there is a correlation between students' perceived stress and the biological marker of stress-cortisol.

- To serve as a working example of research design to physical therapy students starting a research course.

- Provide opportunities for multi-disciplinary scholarly activity to tenured and non-tenured faculty.

This paper will focus on only two aspects of the study: the method validation and whether or not the students lose their diurnal variation when challenged with a stressor.

\section{Participants}

After IRB approval was obtained, the entire student body, of the first year physical therapy class, was approached at the beginning of a structured lecture to ask for their participation in the project. These students were in their fourth month $\left(4^{\text {th }}\right)$ of a thirty three (33) month entry-level Doctor of Physical Therapy (DPT) program. All students had a BA or BS degree prior to admission to the program. The study was explained in detail and informed consent was obtained from 50 students. Of these 50 students, 23 completed the research protocol. The students who completed the study were on average $23( \pm 1.21)$ years old; 18 female, 


\section{RESEARCH AND REPORTS}

5 male; 22 Caucasian, 1 Asian, 1 Hispanic with an average undergraduate GPA of $3.54( \pm .27)$ and an average GRE score of $1025( \pm 92.7)$.

\section{Design}

Salivary rather than serum or plasma samples were obtained because levels measured in saliva agree very well with the amount of free cortisol in blood. ${ }^{6}$ Studies have shown that the rate of saliva production does not affect the level of cortisol present. ${ }^{7}$ Using saliva also eliminated the possibility of falsely increased cortisol due to anxiety associated with venipunctures.

Baseline salivary cortisol samples were obtained during the beginning of the first semester at a time that was deemed less academically stressful. Test samples were collected the second semester, one in which they take 36 credits. This heavy course load makes this semester particularly difficult for the students and an ideal time to test how stress influences performance.

Students were given specific instructions to follow (Table 1) for all collections and asked to refrigerate the specimens collected in the evening and bring them to campus the following day. For the baseline samples collected in November 2009, participants were asked to collect $1 \mathrm{~mL}$ of saliva at home between 7:30 and 8:30 pm. The subjects collected their saliva samples by drooling, through a straw, into a $2 \mathrm{~mL}$ polypropylene screwcap tube. The specimens were refrigerated overnight and brought to campus the next morning. Participants collected a morning sample the day after their evening collection between 7:30 and 8:00 am.

Table 1. Instructions for students

\section{Sample collection instructions:}

Do not collect a sample if you have a fever.

Avoid alcohol for 12 hours before sample collection.

Do not eat a major meal within 60 minutes of sample collection.

Avoid dairy products for 20 minutes before sample collection.

Avoid foods with high sugar or acidity or high caffeine content immediately before sample collection.

Rinse mouth with water to remove food residue before sample collection.

Please refrigerate samples overnight.

In February of 2010, the second semester, the same participants were again asked to collect an evening saliva sample at home between 7:30 and 8:30 pm the evening before their 8:00 am kinesiology exam. Many students also collected their morning sample at home and brought it in with them. Specimens were collected Sunday night and Monday morning before the test.

\section{Exclusion criteria}

Students previously diagnosed with a condition related to variation in cortisol such as Cushing's syndrome or Addison's disease ${ }^{1}$ were excluded from the study. In addition, students taking steroid medication either oral, nasal or topical were not enrolled. Students were asked to avoid the following situations that have been shown to affect cortisol levels: excessive exercise, smoking, and food or alcohol intake immediately prior to obtaining a sample. ${ }^{4}$

\section{Salivary cortisol determination}

An enzyme immunoassay method purchased from Salimetrics ${ }^{\oplus}$ was used to determine the salivary cortisol levels. This method was chosen because it used a matrix which accommodated the use of saliva. ${ }^{8}$ Other methods available are designed for serum/plasma but are not validated for saliva. After samples were brought to campus, they were stored at $-20^{\circ} \mathrm{C}$ until assayed. Saliva was thawed, vortexed, and centrifuged at $1500 \mathrm{x} \mathrm{g}$ for 15 minutes to spin down any mucus in the sample. The assay was performed by following the package insert from Salimetrics ${ }^{\circledR}$ (State College, PA). ${ }^{8}$ A BIO-TEK, Inc. ELx800 Absorbance Microplate Reader, set at 450 $\mathrm{nm}$ wavelength, was used to perform the cortisol assays in the MLS student chemistry laboratory. Raw data (absorbance values) was sent to Salimetrics for interpretation using Gen $5^{\mathrm{TM}}$ data analysis software, a program that was not installed on our instrument at the time.

\section{Method Verification}

Ninety-one salivary cortisol samples from participants were assayed in-house as previously described. Aliquots of these samples were also sent, on dry-ice, to Salimetrics for correlation.

Statistical analysis was performed using SPSS 18.0 PASW Statistics. A paired sample t-test and Pearson correlation was performed $(\mathrm{n}=91)$ on the split samples performed in the student lab and sent to Salimetrics. The paired sample correlation $r=.915$ and $\mathrm{p}<.001$ 


\section{RESEARCH AND REPORTS}

showed a very strong correlation between the two methods (Figure 1).

An intra-assay coefficient of variation was 5.5\%.

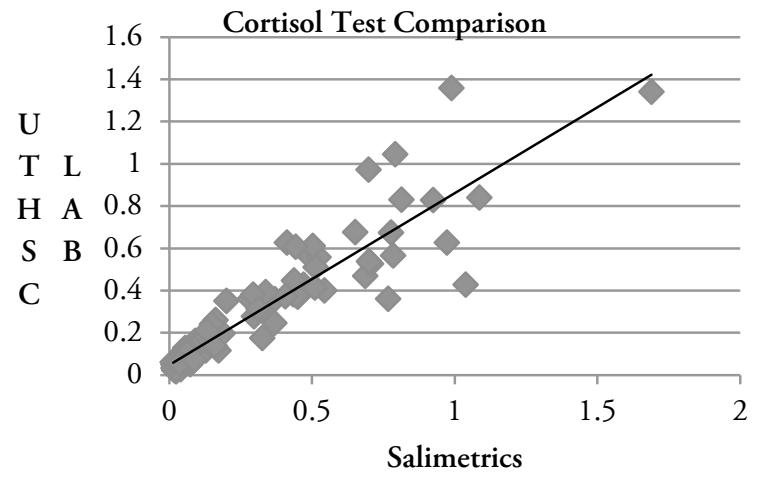

Figure 1. Method Verification - Ninety-one salivary cortisol samples were assayed at UTHSC and at Salimetrics.

A laboratory offering the salivary cortisol testing for clinical purposes would be required to perform a more in-depth verification protocol. Sensitivity, specificity and lower limit of detection assays were not performed.

\section{RESULTS}

Interpretation of the students' cortisol values $(n=23)$ showed a mean fall evening value of $.075 \mathrm{ug} / \mathrm{dL}$, a mean fall morning value of $0.63 \mathrm{ug} / \mathrm{dL}$, a mean test evening value of $0.08 \mathrm{ug} / \mathrm{dL}$, and a mean test morning value of $0.41 \mathrm{ug} / \mathrm{dL}$ (Table 2). Ranges are shown on Table 3. The normal diurnal variation between evening and morning samples did occur with evening samples reading lower than morning samples.

A paired samples t-test comparing the difference between the evening and morning values collected in the fall (baseline), compared with the difference between the evening and morning values collected in February (test) showed a statistically significant difference $(p=.028)$. There was a greater difference in the evening and morning values during the non-stressful

Table 2. Salivary cortisol values*

\begin{tabular}{|c|c|c|c|c|c|}
\hline & $\mathrm{N}$ & Mean & Std. deviation & $\begin{array}{c}\text { Expected value } \\
\text { males } \\
\text { age } 21-308\end{array}$ & $\begin{array}{c}\text { Expected } \\
\text { value females } \\
\text { age } 21-308\end{array}$ \\
\hline Baseline & & & & & \\
\hline $\begin{array}{l}\text { Fall evening } \\
\text { Baseline }\end{array}$ & 23 & 0.075 & 0.05955 & ND-.308 & ND- 0.7403 \\
\hline $\begin{array}{l}\text { Fall morning } \\
\text { Test }\end{array}$ & 23 & 0.6300 & 0.033694 & $0.112-0.743$ & $0.272-1.348$ \\
\hline $\begin{array}{l}\text { Spring evening } \\
\text { Test }\end{array}$ & 23 & 0.0809 & 0.11955 & ND-.308 & ND- 0.7403 \\
\hline Spring morning & 23 & 0.4117 & 0.26819 & $0.112-0.743$ & $0.272-1.348$ \\
\hline
\end{tabular}

*All values are reported in $\mathrm{ug} / \mathrm{dL}$.

Table 3. Salivary cortisol ranges*

\begin{tabular}{lcccc}
\hline & Fall evening & Fall Morning & Test Evening & Test Morning \\
Range & .19 & 1.47 & .47 & .95 \\
Minimum & .01 & .19 & .02 & .04 \\
Maximum & .20 & 1.65 & .49 & .99 \\
\hline
\end{tabular}

*All values are reported in ug/dL.

semester (mean $=.5557$ ), than the difference in the evening and morning values during the test semester $($ mean $=.3291)$. Cohen's d effect size $=.4$. Interestingly, this difference was due to lower cortisol values on the morning of the test than on the morning they were collected in the fall. A paired samples t-test comparing the difference in the baseline morning cortisol value $($ mean $=0.6300)$ and test morning cortisol $($ mean $=$
0.4117 ) showed a statistically significant difference $\mathrm{p}=$ .019 . Results for the paired samples t-tests are shown in Table 4.

Power analysis was not performed with this pilot study because estimates of effect size and sample variance must be known before conducting a power analysis. ${ }^{9,10}$ When larger studies are performed, researchers can 


\section{RESEARCH AND REPORTS}

utilize effect size estimates from pilot data such as the current study. Cohen's d effect size was calculated by subtracting baseline diurnal differences from test diurnal differences and dividing by the pooled estimate of variance.

Table 4. Paired samples t-test

\begin{tabular}{lllc}
\hline & & $\mathrm{df}$ & Sig. (2-tailed) \\
Pair 1 & fall difference- test difference & 22 & $.028^{*}$ \\
Pair 2 & fall evening-test evening & 22 & .826 \\
Pair 3 & fall morning-test morning & 22 & $.019^{*}$ \\
\hline
\end{tabular}

*denotes statistical significance, $\mathrm{p}<.05$

\section{DISCUSSION}

People are able to maintain relative homeostasis because the human body naturally responds to stressful situations in ways that allow us to react appropriately. Dealing with stress for prolonged periods of time will eventually affect us adversely. ${ }^{11}$ Students deal with the stress of school individually with some thriving but others struggling to cope. Helping students learn how to cope with academic pressures benefits all involved.

In this group of students, the cortisol level prior to the stressful event did not increase as expected. This may be due to the fact that the students had an additional day to study due to weather related closing of the campus. Another explanation might be that these students have been coping with this type of stressor for the last four or five years resulting in desensitization. Repeated exposure to the same stressor can result in desensitization or failure to respond. This is called adaptation or habituation. ${ }^{11}$ Another explanation could be that these students as a group have developed skills that allow them to manage test related stress. The students are selected through a competitive process using criteria that would exclude students who succumb easily to the stress of an exam.

This initial small sample study made the researchers aware of problems that should be addressed before a larger study is designed. When participants bring in specimens collected at home, investigators need to make sure the specimen is properly labeled before the participant leaves. Several participants were dropped from the study because the collection tubes were not labeled. One sample was lost because the cap was not tightened properly and the specimen leaked. One participant's data was deleted from the study because her fall evening value was abnormally high and inconsistent with the rest of her results. This may have been due to a collection error, medication, or a personal issue.

Cortisol diurnal variation was not lost in the study participants, but further studies should be performed due to the low percentage of students completing the study and the lack of demographic diversity. The weekend immediately before the test, the students had an unexpected 3-day weekend due to a weather- related closure of the university. It is possible that this extra day to study decreased the stress the students would have otherwise felt. Also, these physical therapy students tend to be more physically active, compared to a more generalized adult population, which could affect the results.

\section{CONCLUSIONS}

Diurnal variation of cortisol was not lost in the study participants but further studies need to be performed due to the low number of participants completing the study and the lack of demographic diversity. Other variables that should be controlled, in future studies, include: physical activity level, grade point average, and body mass index (BMI).

Even though the method validation performed in the student laboratory setting demonstrates that it is indeed possible to obtain the same excellent correlation as is seen in a clinical setting, the student laboratory is not CLIA certified so assays can be performed for research use only.

The fact that the students' cortisol levels were significantly higher in the fall semester than the spring semester has raised some interesting questions that will be investigated in the future. One such question is, "Does the stress of life events, including moving to a new location, affect students more than academic stressors"?

ACKNOWLEDGEMENTS The authors would like to thank Thomas A. Hughes M.D. at the University of Tennessee Health Science Center for his advice on this project. Kametricia N. Derricks is gratefully acknowledged for her contributions. This project was supported with a seed grant from the College of Allied 


\section{RESEARCH AND REPORTS}

Health Sciences at the University of Tennessee Health Science Center.

\section{REFERENCES}

1. Burtis CA, Ashwood, E.R., Bruns. D.E. Tietz Textbook of Clinical Chemistry and Molecular Diagnostics, 4th ed. St. Louis: Elsevier Saunders; 2006.

2. Kalman B, Grahn R. Measuring Salivary Cortisol in the Behavioral Neuroscience Laboratory. The Journal of Undergraduate Neuroscience 2004;2:A41-9.

3. Kudielka B, Kirschbam C. Sex differences in HPA axis responses to stress: a review. Biological Psychology 2005;69:113-32.

4. Smeets T, Dziobek I, Wolf O. Social cognition under stress: Differential effects of stress-induced cortisol elevations in healthy young men and women. Hormones and Behavior 2009;55:507-13.

5. Luethi M, Meier B, Sandi C. Stress effects on working memory, explicit memory, and implicit memory for neutral and emotional stimuli in healthy men. Frontiers in Behavioral
Neuroscience. Available from: http://www.frontiersin.org/ behavioral_neuroscience/10.3389/neuro.08.005.2008/full. Jan, 2009 [cited Jan 14, 2011].

6. Arafah B, Nishiyama F, Tlygeh H, Hejal R. Measurement of Salivary Cortisol Concentration in the Assessment of Adrenal Function in Critically Ill Subjects: A Surrogate marker of the Circulating Free Cortisol. J Clin Endocrinol Metab 2007;92:2965-71

7. Vining R, McGinley R. The measurement of hormones in saliva: Possibilities and pitfalls. J Steroid Biochem. 1987;27:8194.

8. Salimetrics ${ }^{\mathrm{TM}}$. High Sensitivity Salivary Cortisol Enzyme Immunoassay Kit. July 2009. State College, PA.

9. Statistical Computing Seminars: Introduction to Power Analysis. Available from: http://www.ats.ucla.edu/stat/ seminars/intro_power/default.htm [cited March 30, 2011].

10. Lenth, R.V., Statistical Power Calculations, J. Anim. Sci. 2007;85:E24-9.

11. Miller D, O'Callaghan J. Neuroendocrine Aspects of the Response to Stress. Metabolism 2002;51:5-10.

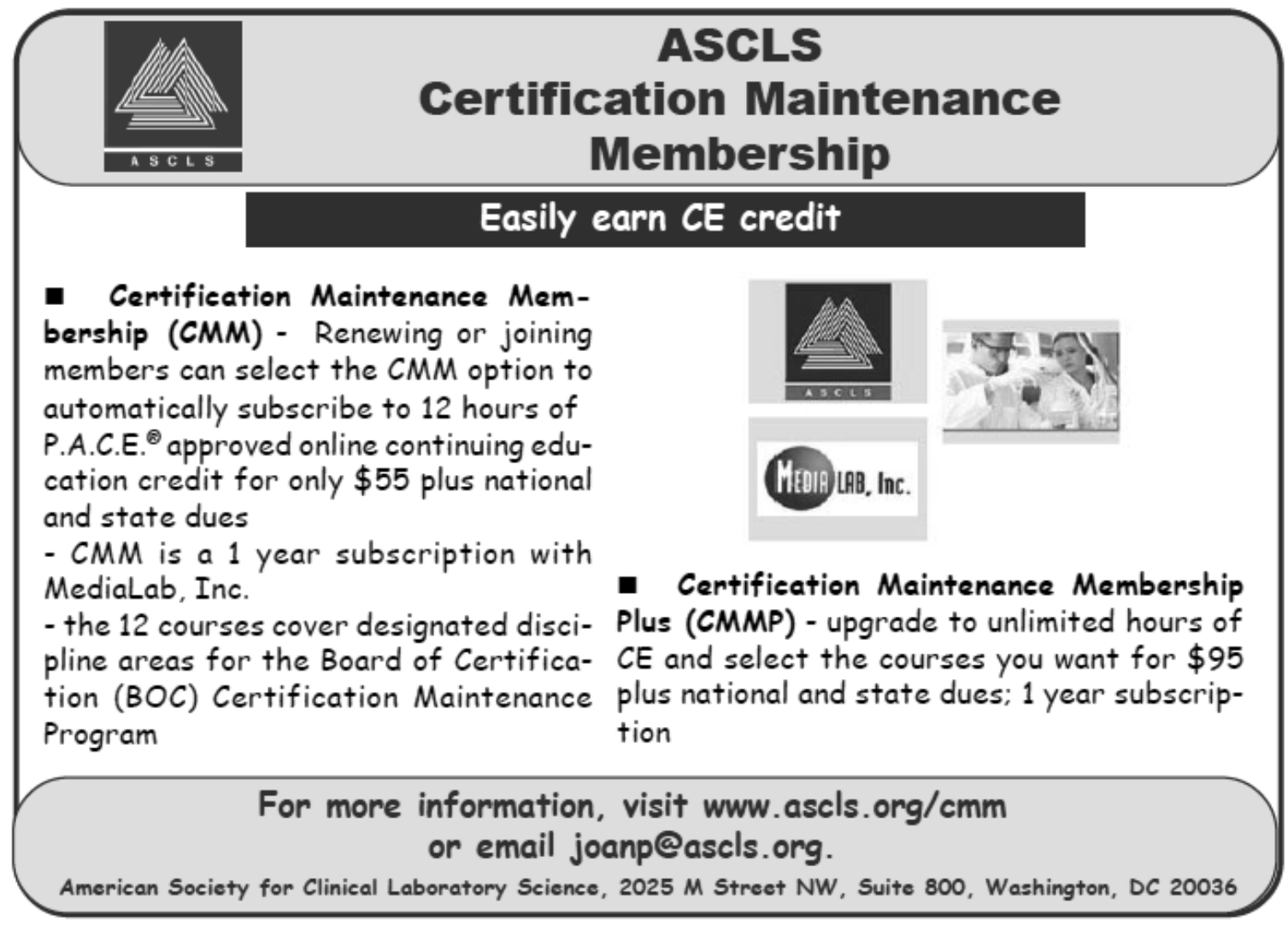

